\title{
Estudio Cinético de la Descomposición Térmica de Tres Derivados del Pirano $\left(\mathrm{C}_{5} \mathrm{H}_{6} \mathrm{O}\right)$
}

\author{
Jennifer J. Lafont ${ }^{1^{*}}$, Manuel S. Páez ${ }^{1}$ y Armando Alvis ${ }^{2}$ \\ Universidad de Córdoba, (1) Facultad de Ciencias Básicas e Ingeniería, Departamento de Química, \\ (2) Facultad de Ciencias Agrícolas, Departamento de Ingeniería de Alimentos, Carrera 6 No 76-103, \\ Km 3, vía Cereté, Córdoba-Colombia (e-mail: jenniferlafontm@yahoo.es)
}

*autor a quien debe ser dirigida la correspondencia

\begin{abstract}
Resumen
El artículo presenta el estudio cinético de descomposición térmica de tres compuestos derivados del pirano que no han sido reportados en la literatura. Estos compuestos son ampliamente utilizados en farmacología y en la obtención de importantes productos a nivel tecnológico e industrial. Los compuestos 2,6-dietoxitetrahidro-2H-pirano; 2-etoxi-6-propoxitetrahidro-2H-pirano y 2-etoxi-6-butoxitetrahidro-2H-pirano se escogieron variando el sustituyente ubicado en la posición 6 del núcleo. Cada experimento cinético fue realizado a cinco temperaturas con intervalos de 10 grados en un rango entre 333 a $373 \mathrm{~K}$, utilizando un sistema de reacción a microescala. Se obtuvieron los productos 2-etoxi-6-hidroxi-tetrahidro-2H-pirano y etileno, propeno, o buteno para cada caso, fueron identificados mediante la técnica de cromatografía de gases acoplada a masas. Las reacciones resultaron ser unimoleculares homogéneas y de primer orden. Teniendo en cuenta los parámetros cinéticos y termodinámicos obtenidos, se puede decir que la reacción es compatible con un estado de transición cíclico de seis miembros, concertado y semipolar.
\end{abstract}

\section{Kinetic Study of the Thermal Decomposition of Three Derived from the Pyran $\left(\mathrm{C}_{5} \mathrm{H}_{6} \mathrm{O}\right)$}

\begin{abstract}
The article presents a kinetic study of the thermal decomposition of three compounds derived from pyran that have not been reported in the literature. These compounds are widely used in pharmacology to obtain important products of technological and industrial interest. The compound (2,6-dietoxitetrahydro-2H-pyran; 2-etoxi-6-propoxytetrahydro-2H-pyran and 2-etoxi-6-butoxytetrahydro-2H-pyran) were chosen by varying the substituent located in the 6 position of the nucleus. Each experiment was done at five temperature with intervals of 10 degrees in a range between 333 and $373 \mathrm{~K}$, using a microscale reaction system. The products obtained, 2-etoxy-6-hydroxytetrahydro- $2 \mathrm{H}$-pyran and ethylene, propene, or butene for each case, were identified using gas chromatography connected to masses. The reactions turned out to be unimolecular, homogeneous and of first order. Considering the kinetic and thermodynamic parameters obtained, it is possible to say that the reaction is compatible with a concerted six membered semi-polar cyclic transition state.
\end{abstract}

Keywords: thermal decomposition, kinetics, pyran, thermodynamic, reaction mechanism 


\section{INTRODUCCIÓN}

El pirano es un compuesto orgánico heterocíclico de seis miembros que incluye en su estructura un átomo de oxígeno, dos insaturaciones y un carbono saturado que no permite la aromaticidad, por lo cual es inestable; sin embargo sus derivados con un doble enlace en el núcleo (dihidropiranos) o totalmente saturados (tetrahidropiranos) son estables y poseen grandes aplicabilidades de tipo biológico, entre las cuales se pueden destacar: la actividad antimicrobial contra las bacterias Gram positiva, fungi, Staphylococcus aureus, Entrococcus faecalis, Escherichia coli, entre otras (Rafat y Hanaa, 2008; Dilsiz et al., 2003; Alam et al., 2004); posee actividad antiviral contra el virus BK, patógeno importante en trasplantes renales (Randhawa et al., 2007), actividad anticonvulsionante que poseen las bases de Mannich con grupos piperazina en el alomaltol (5-hidroxi-2-metil-4H-piran4-ona) y sus derivados (Dilsiz y Çalış. 2007), actividad antihipertensiva (Yuan et al., 2005), actividad hepatoprotectora (Kumar et al., 2003), también son utilizados para el tratamiento del asma, las alergias y en las plantas como herbicidas (Fateme et al., 2002). Además pueden actuar como solventes, reactivos o grupos protectores en síntesis orgánicas para llegar a productos de interés científico, tecnológico e industrial (Baker et al., 2005; Diez et al., 2006).

Debido a la importancia que han demostrado estos compuestos; se consideró pertinente explorar una nueva serie de tres tetrahidropiranos y realizar el estudio cinético de la descomposición térmica de cada compuesto en fase liquida, en los cuales se varía el sustituyente situado en el carbono seis incrementándolo en un átomo de carbono; con el fin de calcular los parámetros termodinámicos, analizar su comportamiento y finalmente proponer un mecanismo de reacción razonable.

Además de la cinética que provee información importante acerca de las velocidades de reacción; las características estructurales de acetales cíclicos que poseen los tetrahidropiranos ensayados, abre otro campo de estudio que ha sido poco explorado desde el punto de vista químico e industrial; debido a que los compuestos acetálicos de cadena abierta son ampliamente utilizados en la industria de polímeros.

\section{METODOLOGÍA}

Los compuestos 2,6-dietoxitetrahidro-2H-pirano; 2-etoxi-6-propoxitetrahidro-2H-pirano y 2-etoxi-6butoxitetrahidro-2H-pirano fueron sintetizados en un trabajo anterior utilizando el siguiente procedimiento:

Síntesis del 2,6-dietoxitetrahidro-2H-pirano: En un reactor sumergido en un baño de hielo se agregó $(0,05 \mathrm{~mol} ; 6,7 \mathrm{ml})$ de 2-etoxi-3,4-dihidro-2H-pirano y $(0.05 \mathrm{~mol} ; 2,9 \mathrm{ml})$ de etanol en cantidades equimolares agitando levemente, luego se agregaron 2 gotas $(0,08 \mathrm{ml})$ de ácido clorhídrico como catalizador, observándose un aumento en la temperatura dejando en reposo la reacción por un tiempo de 4 horas. Posteriormente se adicionó $(1 \mathrm{~g})$ de hidróxido de sodio y se agitó vigorosamente; el producto obtenido se filtró, se secó utilizando $\mathrm{CaCl}_{2}$, se volvió a filtrar y se destiló a presión reducida. Obteniendo un rendimiento del $86 \%$.

Síntesis del 2-etoxi-6-propoxi-tetrahidro-2H-pirano y el 2-etoxi-6-butoxi-tetrahidro-2H-pirano: Se utilizó el procedimiento descrito anteriormente, manteniendo las cantidades equimolares del 2-etoxi3,4-dihidro-2H-pirano $(0.05 \mathrm{~mol} ; 2,9 \mathrm{ml})$ y el alcohol, en este caso $(0.05 \mathrm{ml} ; 3,76 \mathrm{ml})$ de propanol y $(0,05 \mathrm{ml} ; 4,57 \mathrm{ml})$ de butanol. Los rendimientos de las reacciones fueron del $82 \%$ y $78 \%$ respectivamente.

Los productos fueron obtenidos con purezas del $90 \%$ verificadas con un equipo de CG-MS Agilent $6890 \mathrm{~N}$, con detector selectivo de masas Agilent 5973N, e inyector Agilent 7683N DB-Wax 0,25mm x $30 \mathrm{~m}$ longitud nominal $\times 0,25 \mu \mathrm{m}$ espesor de la película.

La termólisis de los compuestos en estudio fue realizada en un minireactor químico que posee un horno cilíndrico recubierto de asbesto y material refractario, cuyo diámetro y longitud son 5,5 y $8,0 \mathrm{~cm}$ respectivamente; en la parte superior posee 20 perforaciones para introducir las ampollas de vidrio 
con la sustancia a termolizar, tiene además sensor de temperatura, termocupla tipo $\mathrm{K}$, controlador Barber-Colman, tipo PID, con una incertidumbre de $\pm 1 \mathrm{~K}$.

Los productos de termólisis fueron identificados por GC-MS y sus espectros fueron comparados con los de literatura, resultando estar en buen acuerdo.

\section{Cinética}

Para realizar la cinética del compuesto 2,6-dietoxitetrahidro-2H-pirano; se inyectó 2,5 $\mu \mathrm{L}$ del mismo en cada ampolla de vidrio, en total seis por serie, luego fueron selladas y colocadas en el sistema térmico a una temperatura previamente calibrada en $333,15 \mathrm{~K}$ y se fueron retirando a cada 60 minutos. Posteriormente cada ampolleta fue enfriada a 273,15K para detener la reacción. Las muestras termolizadas fueron preparadas y examinadas en un cromatógrafo de gases-masas; usando acetona como solvente (mejor resolución), también se ensayó con acetato de etilo y acetonitrilo, pero los resultados no fueron satisfactorios. Este procedimiento se repitió de 3 a 4 veces para cada temperatura de estudio a intervalos de diez grados en un rango de 333,15 y $373,15 \mathrm{~K}$. El procedimiento anterior fue el mismo para los compuestos 2-etoxi-6-propoxitetrahidro-2H-pirano y 2etoxi-6-butoxitetrahidro-2H-pirano.

Se observó que al aumentar la temperatura la reacción se hace mas rápida, de ahí que el tiempo de reacción para sacar las ampolletas del sistema debe ser cada vez menor, así: 60, 45, 30, 22,5 y 15 minutos respectivamente.

Las muestras termolizadas fueron inyectadas en el cromatógrafo de gases-masas del cual fueron obtenidos los valores de las áreas de descomposición del reactivo con respecto al tiempo para cada temperatura de estudio.

\section{Tratamiento de datos y cálculo de parámetros termodinámicos}

Los valores de las áreas $a$ de la descomposición térmica de cada derivado piránico fueron sustituidas por la propiedad física $\lambda_{0}$ y $\lambda_{t}$; donde $\lambda_{0}$ representa la concentración inicial del reactivo a $\mathrm{t}=0 \mathrm{y}$ $\lambda_{t}=\left(a_{0}-x\right)$ que representa la disminución de la concentración del reactivo piranico a medida que transcurre el tiempo; como se define en la siguiente ecuación (Laidler y Meiser, 1998):

$\left(a_{0}-x\right)=a_{0} e^{-k t}$

$\lambda_{t}=\lambda_{0} e^{-k z}$

Aplicando Ln a ambos términos:

$\ln \lambda_{t}=\ln \lambda_{0}-k t$

La cuantificación de la constante de velocidad experimental $k$ se obtuvo de las pendientes de los gráficos correspondientes a $\ln \lambda_{\varepsilon}$ vs. t, para cada temperatura de estudio.

Los datos de las constantes obtenidas se ajustaron a la expresión de Arrhenius:

$k=A e^{-\frac{E_{\mathrm{n}}}{R T}}$

La forma logarítmica de esta ecuación es:

$\ln k=-\frac{E_{a}}{R T}+\ln A$

Al realizar la representación gráfica de $\ln \xi$ : vs $T^{-1}$ de cada compuesto se obtuvo una línea recta con pendiente igual a $-E_{a} / R$ y un intercepto correspondiente al $\ln A$; con estos valores fueron calculados 
los parámetros termodinámicos, teniendo en cuenta la ecuación de Eyring, que describe el factor pre-exponencial para reacciones unimoleculares en solución:

$A=e^{\frac{k_{g} T}{h}}=e^{\frac{\Delta S^{*}}{R}}$

\section{RESULTADOS Y DISCUSIÓN}

Todas las gráficas de In $\lambda_{z}$ vs. $t$ para cada temperatura ensayada en los tres compuestos de estudio, presentaron buena correlación lineal, lo cual confirma que las reacciones cumplen con una ecuación cinética de primer orden.

En general los valores de las constantes de velocidad $k$ presentan proporcionalidad con la temperatura; en este estudio cinético se comprueba que al incrementar la temperatura diez grados, la velocidad de la reacción aumenta, este comportamiento se observó para los tres compuestos. Los valores de las constantes de velocidad para cada temperatura, se reportan en la tabla 1.

Al comparar las características estructurales de los sustituyentes de la serie de compuestos estudiados, se observó que al aumentar la complejidad del sustituyente en un átomo de carbono, disminuyen los valores de las constantes de velocidad, haciendo más lenta la reacción; de esta forma se comprueba que al aumentar el impedimento estérico en la molécula la velocidad de la reacción disminuye.

A partir de los valores de la tabla 1 se obtuvieron las graficas de Arrhenius en las cuales se relacionan los valores de la constantes de velocidad con respecto a las temperaturas ensayadas figuras 1-3 presentando buena correlación para los compuestos 2,6-dietoxitetrahidro-2H-pirano; 2etoxi-6-propoxitetrahidro-2H-pirano y 2-etoxi-6-butoxitetrahidro-2H-pirano.

En la tabla 2 se describen los resultados de los parámetros termodinámicos calculados a la temperatura de $353,15 \mathrm{~K}$ para los tres compuestos estudiados así: para el 2,6-dietoxitetrahidro- $2 \mathrm{H}$ pirano se referencia como $\mathrm{N}^{\circ} 1$; el 2-etoxi-6-propoxitetrahidro-2H-pirano como $\mathrm{N}^{\circ} 2$ y el 2-etoxi-6butoxitetrahidro-2H-pirano como $\mathrm{N}^{\circ} 3$.

De acuerdo con los valores de esta tabla, las tres reacciones de descomposición térmica se pueden interpretar en forma similar; los valores negativos de la entropía de activación $\left(\Delta S^{\neq}\right)$indican que el estado de transición presenta menor valor con respecto al del reactivo, disminuyendo así los grados de libertad; es decir que el estado de transición es mas ordenado que los reactivos.

Los valores positivos de entalpía de activación $\left(\Delta \mathrm{H}^{\ddagger}\right)$ indican que estas reacciones son endotérmicas; los valores positivos de la energía libre de activación $\left(\Delta \mathrm{G}^{\neq}\right)$indican que las reacciones son estables, no espontáneas, endergónicas y que estos sistemas no poseen suficiente energía interna para que ocurran las reacciones químicas.

El valor de la energía de activación $E_{a}$ es muy similar al cambio en entalpía; lo cual cumple con la expresión $E_{a}=\Delta \mathrm{H}^{\ddagger}+\mathrm{n} R T$. El valor del factor pre-exponencial $\mathrm{A}$ fue obtenido en el rango de $10^{11}$ $10^{13} \mathrm{~s}^{-1}$, el cual está dentro del intervalo reportado en literatura para reacciones de primer orden (Zapata et al., 2006; Julio et al., 2007).

De acuerdo con los valores obtenidos de los parámetros termodinámicos y productos finales identificados en las reacciones de descomposición térmica de los compuestos 2,6-dietoxitetrahidro$2 \mathrm{H}$-pirano; 2-etoxi-6-propoxitetrahidro-2H-pirano y 2-etoxi-6-butoxitetrahidro-2H-pirano; se propone un mecanismo que involucra un estado de transición cíclico de seis miembros, semipolar y concertado, en el que posteriormente ocurre una reorganización para formar el producto 2-etoxi-6hidroxitetrahidro-2H-pirano y como subproducto etileno, propeno o buteno respectivamente, véase figura 4. 
Tabla 1: Valores de las constantes de velocidad para los compuestos de estudio a diferentes temperaturas.

\begin{tabular}{|c|c|c|c|c|c|}
\hline $\mathbf{N}^{\circ}$ & Compuesto & $\mathbf{T}(\mathrm{K})$ & $k\left(s^{-1}\right)$ & $\operatorname{Ln} k / \mathrm{s}^{-1}$ & $1 / \mathrm{T} \times 10^{3} / \mathrm{K}^{-1}$ \\
\hline 1 & 2,6-dietoxitetrahidro-2H-pirano & $\begin{array}{l}333,15 \\
343,15 \\
353,15 \\
363,15 \\
373,15\end{array}$ & $\begin{array}{l}3,56 \text { E-06 } \pm 0,07 \\
1,37 \text { E-05 } \pm 0,04 \\
4,08 \text { E-05 } \pm 0,16 \\
1,23 \text { E-04 } \pm 0,22 \\
3,48 \text { E-04 } \pm 0,01\end{array}$ & $\begin{array}{l}-12,5450 \\
-11,1910 \\
-10,1050 \\
-9,0040 \\
-7,9620\end{array}$ & $\begin{array}{r}3,001 \\
2,914 \\
2,831 \\
2,7536 \\
2,679\end{array}$ \\
\hline 2 & $\begin{array}{l}\text { 2-etoxi-6-propoxitetrahidro-2H- } \\
\text { pirano }\end{array}$ & $\begin{array}{l}333,15 \\
343,15 \\
353,15 \\
363,15 \\
373,15\end{array}$ & $\begin{array}{l}1,58 \mathrm{E}-08 \pm 0,05 \\
7,29 \mathrm{E}-08 \pm 0,12 \\
2,43 \mathrm{E}-07 \pm 0,32 \\
8,34 \mathrm{E}-07 \pm 0,02 \\
2,68 \mathrm{E}-06 \pm 0,20\end{array}$ & $\begin{array}{l}-17,9640 \\
-16,4336 \\
-15,2302 \\
-13,9970 \\
-12,8296\end{array}$ & $\begin{array}{l}3,001 \\
2,914 \\
2,831 \\
2,7536 \\
2,679\end{array}$ \\
\hline 3 & $\begin{array}{l}\text { 2-etoxi-6-butoxitetrahidro-2H- } \\
\text { pirano }\end{array}$ & $\begin{array}{l}333,15 \\
343,15 \\
353,15 \\
363,15 \\
373,15\end{array}$ & $\begin{array}{l}\text { 1,38E-11 } \pm 0,03 \\
7,05 \mathrm{E}-11 \pm 0,51 \\
3,44 \mathrm{E}-10 \pm 0,08 \\
1,26 \mathrm{E}-09 \pm 0,01 \\
4,64 \mathrm{E}-09 \pm 0,12\end{array}$ & $\begin{array}{l}-25,0009 \\
-23,3751 \\
-21,7911 \\
-20,4921 \\
-19,1888\end{array}$ & $\begin{array}{l}3,001 \\
2,914 \\
2,831 \\
2,7536 \\
2,679\end{array}$ \\
\hline
\end{tabular}

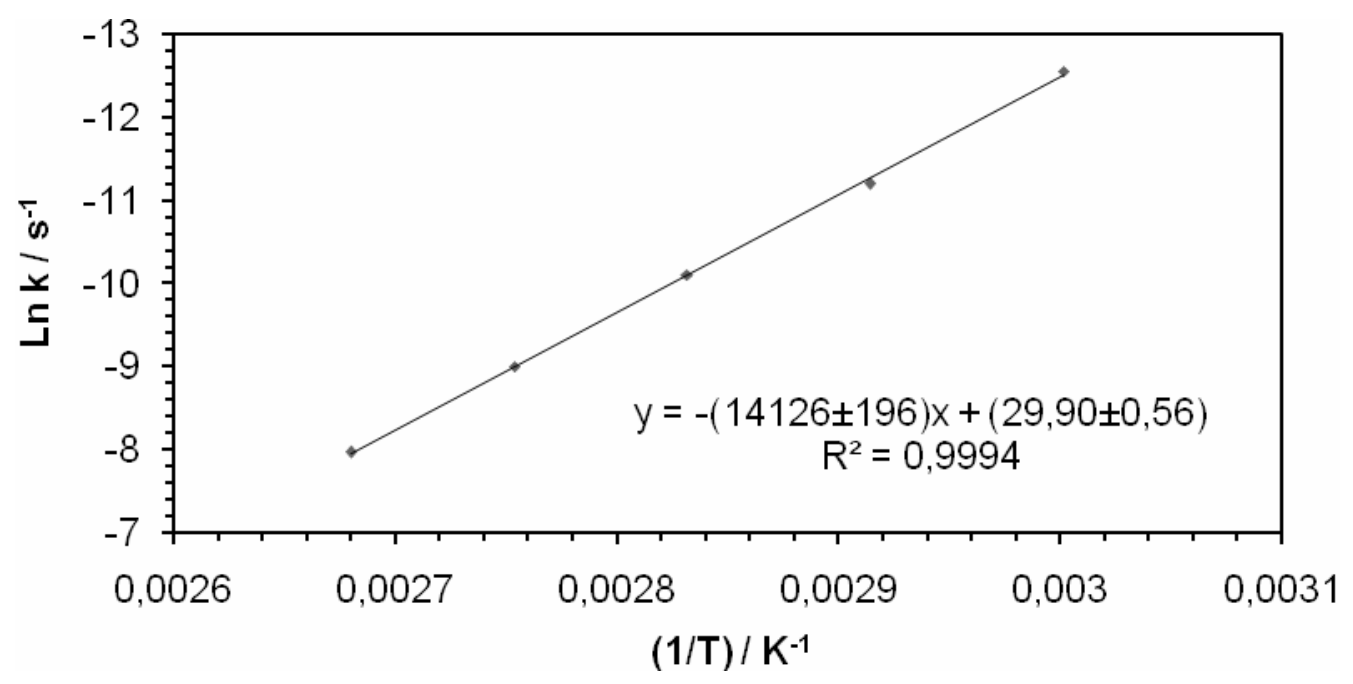

Fig. 1: Gráfico de Arrhenius del 2,6-dietoxitetrahidro-2H-pirano. 


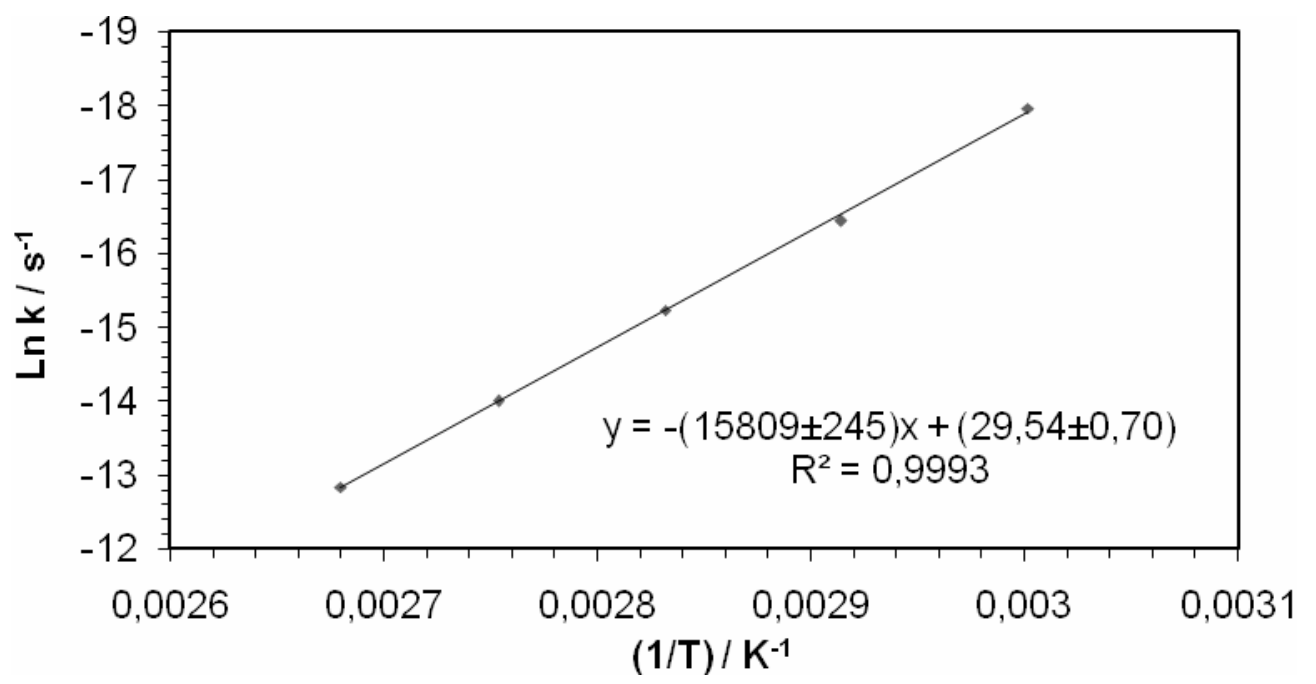

Fig. 2: Gráfico de Arrhenius del 2-etoxi-6-propoxitetrahidro-2H-pirano.

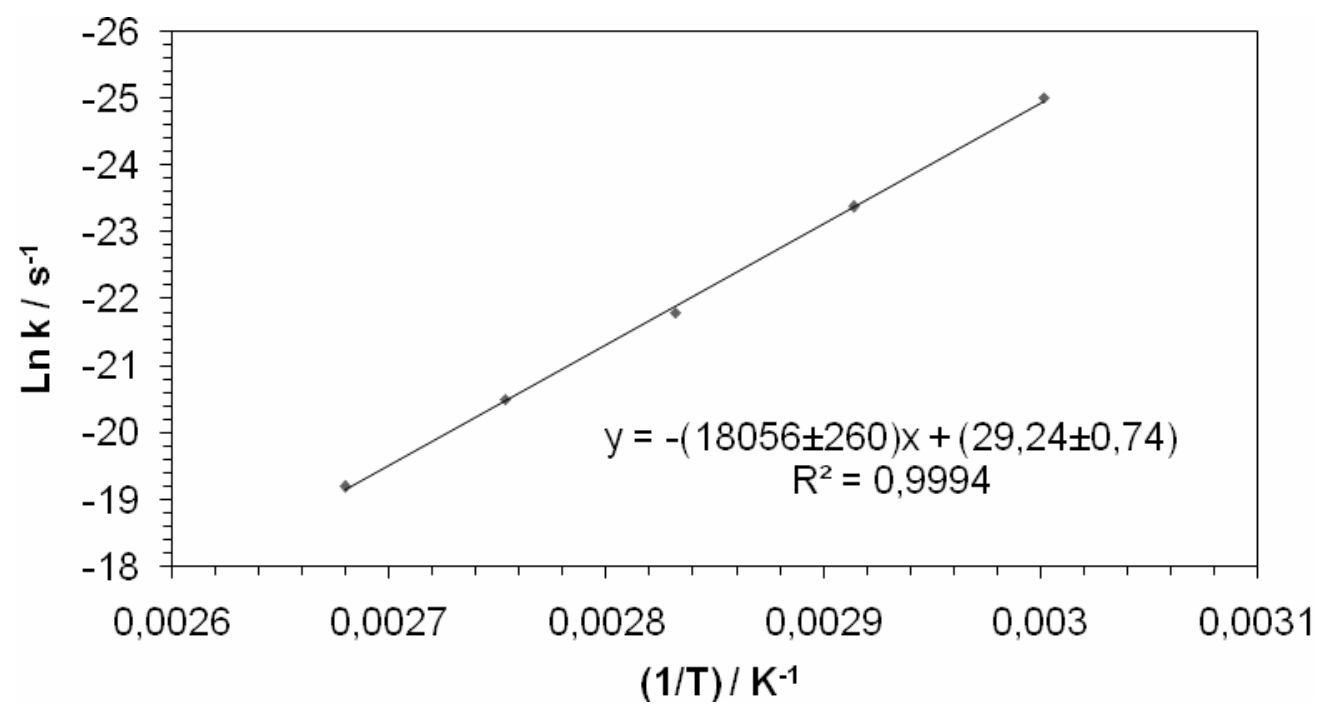

Fig. 3: Gráfico de Arrhenius del 2-etoxi-6-butoxitetrahidro-2H-pirano.

Tabla 2: Parámetros termodinámicos de los compuestos en estudio, calculados a 353,15K.

\begin{tabular}{|c|c|c|c|c|c|c|}
\hline \multirow[t]{2}{*}{$\mathbf{N}^{\circ}$} & \multicolumn{6}{|c|}{ Parámetros } \\
\hline & $\begin{array}{c}k \\
\left(s^{-1}\right)\end{array}$ & $\begin{array}{c}\mathrm{Ea} / \\
\mathrm{KJ}^{-\mathrm{mol}^{-1}}\end{array}$ & $\log _{s^{-1}} A /$ & $\begin{array}{c}\Delta \mathbf{S}^{\ddagger} I \\
\text { J.mol } \\
-1\end{array} \mathbf{K}^{-1}$ & $\begin{array}{c}\Delta \mathrm{H}^{\neq} \mathrm{I}^{-1} \\
\mathrm{KJ} \cdot \mathrm{mol}^{-1}\end{array}$ & $\begin{array}{c}\Delta \mathbf{G}^{\neq} I^{-1} \\
\mathrm{KJ}^{-\mathrm{mol}^{-1}}\end{array}$ \\
\hline 1 & 4,08 E-05 $\pm 0,16$ & $117,44 \pm 3,12$ & $12,98 \pm 0,30$ & $-6,043$ & 114,514 & 116,648 \\
\hline 2 & $2,43 E-07 \pm 0,32$ & $131,44 \pm 1,51$ & $12,83 \pm 0,12$ & $-9,037$ & 128,508 & 131,700 \\
\hline 3 & $3,44 \mathrm{E}-10 \pm 0,08$ & $150,13 \pm 2,64$ & $12,70 \pm 0,27$ & $-11,530$ & 147,200 & 151,272 \\
\hline
\end{tabular}




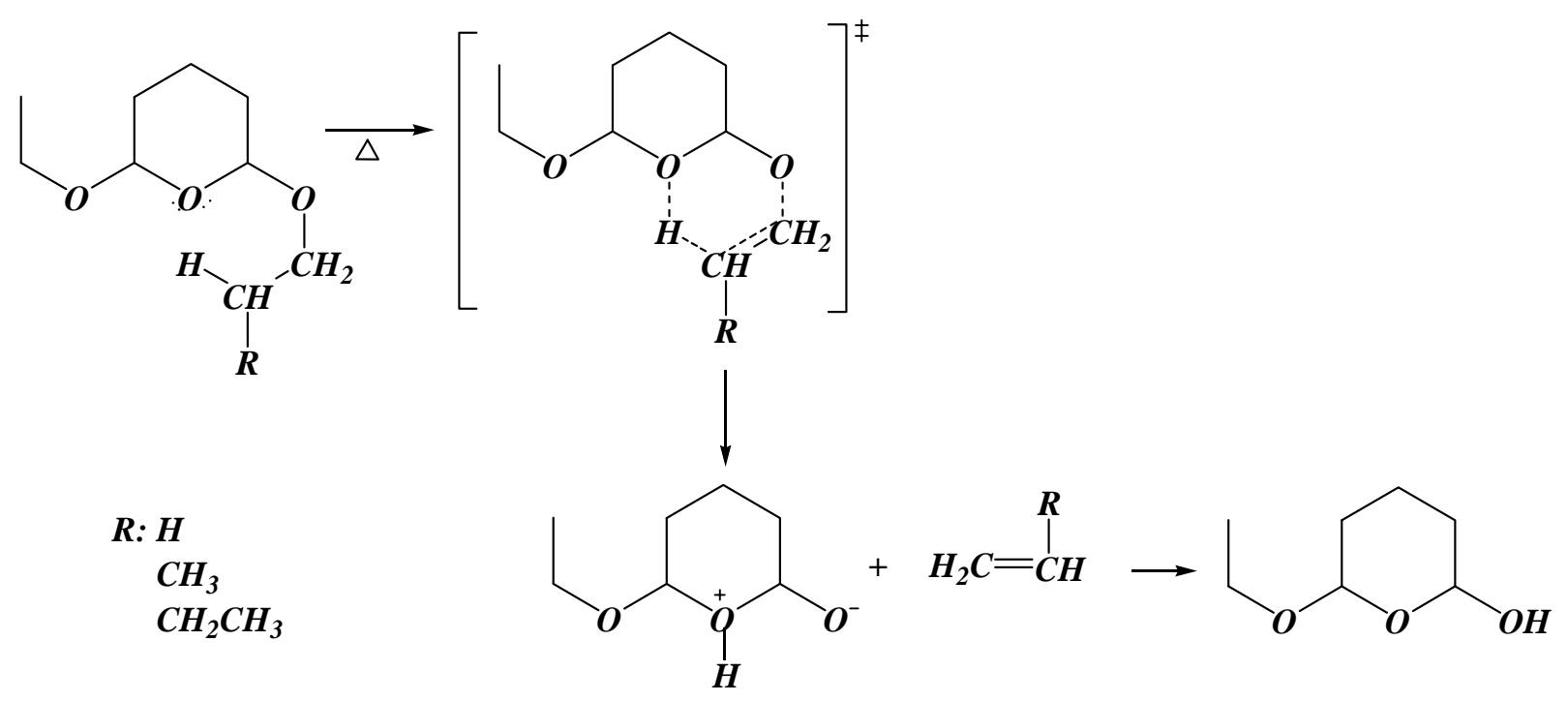

Fig. 4: Mecanismo propuesto para la descomposición térmica de los derivados piránicos.

\section{CONCLUSIONES}

La reacción de descomposición térmica del 2,6-dietoxitetrahidro-2H-pirano; 2-etoxi-6propoxitetrahidro-2H-pirano y 2-etoxi-6-butoxitetrahidro-2H-pirano realizada en el rango de 333,15$373,15 \mathrm{~K}$ siguen una cinética de primer orden. Los valores de las constantes de velocidad para cada reacción mostraron proporcionalidad con las temperaturas de estudio.

Al aumentar la complejidad de los sustituyentes localizados en carbono seis del núcleo piránico, los valores de las constantes de velocidad disminuyen, haciendo más lenta la reacción; concluyendo que el 2-etoxi-6-butoxitetrahidro-2H-pirano presento menor velocidad.

Los parámetros termodinámicos reportados describen reacciones no espontáneas, endergónicas con estado avanzado y menos grados de libertad en los estados de transición con respecto a los reactivos; por lo tanto se propone un estado de transición cíclico de seis miembros, concertado y de carácter semipolar.

\section{AGRADECIMIENTOS}

Al centro de investigaciones de la Universidad de Córdoba por el financiamiento de este proyecto.

\section{REFERENCIAS}

Alam, S., Z. Sarkar y A. Islam; Synthesis and studies of antibacterial activity of pongaglabol, J. Chem. Sci.: 116, 29-32 (2004).

Baker, C. y otros diez autores; Triol protection with 6-benzoyl-3,4-dihydro-(2H)-pyran, Chem. Commun.: 14, 1883-1885 (2005).

Diez, D. y otros seis autores; Synthesis of (R)-2-(Benzyloxy)-tetrahydro-5,5-dimethylfuran by a New Oxidative Rearrangement, Molecules: 11, 959-967 (2006).

Dilsiz, M., D. Demir., R.C. Hider y M. Ozalp; Synthesis and Evaluation of Antimicrobial Activity of New 3-Hydroxy-6-methyl-4-oxo-4H-pyran-2- carboxamide Derivatives, Turk J. Chem.: 27, 757-764 (2003).

Dilsiz, M. y Ü. Çalış; Synthesis of Some Novel Mannich Bases Derived From Allomaltol and Evaluation of Their Anticonvulsant Activities, Hacettepe University Journal of the Faculty of Pharmacy: 27(1), 1-10 (2007). 
Fateme, A., T. Reza., B. Yadollah y S. Aziz; Synthesis of Some Aldoxime Derivatives of 4H-Pyran-4ones, Molecules: 7, 239-244 (2002).

Julio, L. y otros cinco autores; The unimolecular Elimination Kinetics of benzaldoxime in the gas phase, International Journal of Chemical Kinetics: 39, 145-147 (2007).

Kumar, B. y otros cinco autores; Hepatoprotection by 3-bromo-6-(4-chlorophenyl)-4-methylthio-2Hpyran-2-one against experimentally induced liver injury in rats, Acta Pharm.: 53, 91-100 (2003).

Laidler, K.J. y J.H. Meiser; Fisicoquímica, $1^{a}$ Edición 353-366. Compañia editorial continental S.A., Mexico (1998).

Rafat, M.M. y H. Hanaa; Synthesis of progesterone heterocyclic derivatives of potential antimicrobial activity, Acta Pharm: 58, 29-42 (2008).

Randhawa, P., N.A. Farasati y Y. Huang; BK Virus Replication In Vitro: Limited Effect of Drugs Interfering with Viral Uptake and Intracellular Transport, Antimicrobial Agents And Chemotherapy: 51, 4492-4494 (2007).

Yuan, X., X. Fan., X. Zhang., G. Qu y Y. Zhen; An Efficient and Green Synthesis of 5-Oxo-5, 6, 7, 8tetrahydro-4H-benzo-[b]-pyran Derivatives Promoted by $\operatorname{InCl}_{3} \cdot 4 \mathrm{H}_{2} \mathrm{O}$ Under Microwave Irradiation, Chinese Chemical Letters: 16, 886-888 (2005).

Zapata, E., J. Gaviria y J. Quijano, Thermal descomposition of methyl $\beta$-Hydroxyesters in m-xylene solution, International Journal of Chemical kinetics: 39, 92-96 (2007). 